\title{
Study of ultrafast processes in matter by means of time-resolved electron diffraction and microscopy
}

\author{
Sergei Aseyev ${ }^{l}$, Boris Mironov ${ }^{l}$, Sergei Andreyev ${ }^{l}$, Victor Kompanets ${ }^{l}$, Anatolii Ischenko ${ }^{2}$, \\ Igor' Kochikov ${ }^{3}$, Oleg Misochko ${ }^{4}$, Sergei Chekalin ${ }^{1, *}$, and Evgenii Ryabov ${ }^{l}$ \\ ${ }^{1}$ Institute of Spectroscopy, Russian Academy of Sciences, Troitsk, Moscow, 108840 Russia \\ ${ }^{2}$ Moscow Technological University, 86 Vernadskogo ave., Moscow, 119571 Russia \\ ${ }^{3}$ Moscow State University, 1 Leninskie Gory, Moscow, 119991 Russia \\ ${ }^{4}$ Institute of Solid State Physics, Russian Academy of Sciences, Chernogolovka, 142432 Russia
}

\begin{abstract}
One of the most fundamental problems of modern natural science is the direct observation of atomic motions in the course of various processes. For this purpose, in the experiment it is necessary to provide high spatial-temporal resolution. The solution to this problem is achieved by using a pulsed electron beam of ultrashort duration to create a stroboscopic diffraction pattern in the method of time-resolved electron diffraction (TRED). Three types of experimental schemes have been developed at our lab. The experimental complex includes (i) $20-\mathrm{keV}$ table-top apparatus for TRED, (ii) $75-\mathrm{keV}$ ultrafast transmission electron microscope and (iii) lensless table-top device for femtosecond electron diffraction. The obtained experimental results are presented.
\end{abstract}

At present, the study of the structural dynamics of matter with a high spatially-temporal resolution is one of the key topics of modern researches. It is of undoubted interest for condensed matter physics, molecular and chemical physics, biophysics, and materials science [1-3]. Now ultrafast electron microscopy and electron diffraction are rapidly developing fields. These methods are based on probing coherent laser-induced processes in various materials using photoelectron bunches. This approach allows one to supplement the high temporal resolution provided by femtosecond lasers with the atomic spatial resolution, inherent in electron diffraction technique. As a result, one can make a video of the behavior of matter matched in 4D space - time continuum.

The first direct observation of the generation of coherent optical phonons in thin Sb film by TRED has been done using the experimental scheme, depicted in Fig. 1 [3]. The semimetal sample was excited by a femtosecond laser pulse and probed with a pulsed photoelectron beam. The vibrational modes of $\mathrm{Sb}$ lattice have two optical $\mathrm{A}_{1 \mathrm{~g}}$ and $\mathrm{E}_{\mathrm{g}}$ modes and two lowfrequency $\mathrm{A}_{1 \mathrm{u}}$ and $\mathrm{E}_{\mathrm{u}}$ acoustic modes. The fundamental of a femtosecond Ti:Sa laser was used as the pump beam. The pulsed photoelectron beam was formed under the irradiation of

\footnotetext{
*Corresponding author: chekalin@isan.troitsk.ru
} 
the semitransparent silver photocathode by III harmonic of the same Ti:Sa laser and was used as the probe beam. In this manner, the optical and photoelectron pulses were strictly synchronized. The pump energy fluence on the sample was about $1.5 \mathrm{~mJ} / \mathrm{cm}^{2}$.

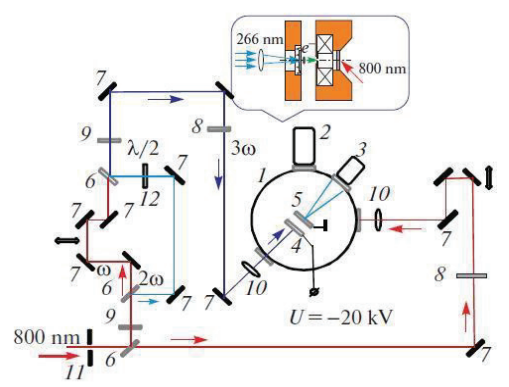

Fig. 1. Schematic of the setup for investigations by TRED with femtosecond resolution: (1) vacuum chamber; (2) turbomolecular pump; (3) position-sensitive detector based on MCP's; (4) silver photocathode; (5) anode + target; (6) laser beam dividers; (7) mirrors; (8) radiation attenuator; (9) converters of laser radiation into the second and third harmonics; (10) lenses; (11) diaphragm; (12) optical rotator. Inset shows the key parts with permanent magnetic lens. Gun-to-sample electron flight path is $12 \mathrm{~mm}$.

In the experiment the variation of the intensity of the diffraction pattern caused by the optical excitation of the sample was studied as a function of the delay time between the pump and probe photoelectron pulses. We observed the sharp decay (with the characteristic time of about $300 \mathrm{fs}$, which corresponded to temporal resolution of the technique) in the diffraction signal after laser irradiation of the $\mathrm{Sb}$ film for positive temporal delay. Besides, the distinct oscillations with the period in sub-picosecond domain were also observed within about 20 ps. The Fourier analysis showed that the observed signal modulation was due to the presence of four distinct modes with frequencies of 1.1, 3.4, 4.6, and 6.4 THz (Fig. 2). Only three of these modes were observed earlier in the experiments on optical probing [4]. Knowing the attribution of the phonon modes in $\mathrm{Sb}$ from the literature, it is possible to associate the frequencies of 4.6 and $3.4 \mathrm{THz}$ with the totally symmetric $\left(A_{1 g}\right)$ and twofold degenerate $\left(E_{g}\right)$ optical phonons and $1.1 \mathrm{THz}$ with the difference vibration $A_{1 g}-E_{g}$. The frequency of $6.4 \mathrm{THz}$ for antimony in the time domain was not observed earlier. It can be associated with the second harmonic $E_{g}$.

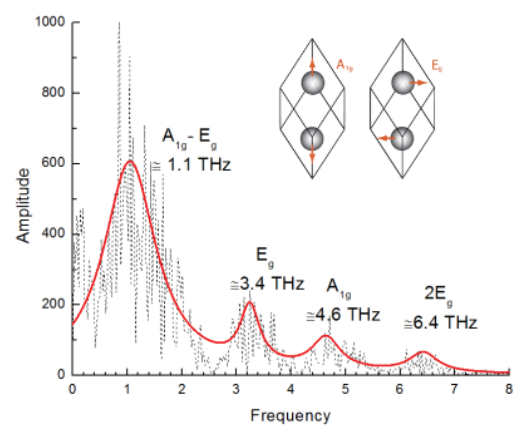

Fig. 2. The Fourier transformation of the normalized intensity of electron diffraction reflections in $\mathrm{Sb}$ film vs time after irradiation by femtosecond laser pulses. The signal modulation is due to the optical phonons illustrated in the inset.

Quite recently, at the Institute for Spectroscopy RAS an ultrafast transmission $75-\mathrm{keV}$ photoelectron microscope was created [5]. The setup is based on commercial transmission electron microscope Hitachi H-300 and is intended for the researches of dynamic processes in the solids by excitation of a sample by femtosecond laser pulses and with probing emerging 
dynamics using $\sim 7$ ps pulsed photoelectron beam. The introduction of an adjustable optical delay between excitation and probing channels enables observation of ultrafast processes in matter. Our apparatus has been combined with $80-\mathrm{MHz}$ femtosecond laser Mai-Tai, 2.5-MHz femtosecond fiber laser Antaus, or 1-kHz source of amplified femtosecond laser pulses with an energy of up to $4 \mathrm{~mJ}$ per laser pulse. In the first part of the experiments it was possible to visualize the interaction of picosecond $75-\mathrm{keV}$ electron beam with charged cloud emitted from copper target under the action of focused femtosecond laser radiation. In the next part, the laser-induced plasma and associated processes in the vicinity of solid target will be investigated with high spatio-temporal resolution.

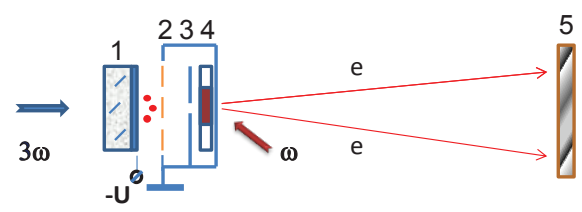

Fig. 3. Schematic diagram of lensless apparatus for TRED: (1) silver photocathode; (2) 0.5-mm-mesh; (3) 100- $\mu$-diaphragm; (4) thin sample; (5) position-sensitive detector. $U$ is accelerating voltage.

A distinguishing feature of the third instrument developed at our lab is the absence of collimating/focusing elements for the electron beam (Fig. 3). Since the planar silver cathode emits photoelectrons with minimal divergence and using a mesh anode further minimizes defocusing, the electron beam reaches the sample with nearly parallel momentum. The completely lensless design dramatically reduces the electron gun-to-sample electron flight path and therefore significantly improves the temporal resolution of TRED [6]. Comparing with the setup, depicted in Fig. 1, the lensless device (Fig. 3) is capable to work with different accelerating voltages. This opens wide opportunities for the experiments.

This study was supported by the Ministry of Education and Science of the Russian Federation (Project No. RFMEFI61316X0054).

\section{References}

[1] A.A. Ischenko, G.V. Girichev, Yu.I. Tarasov, Electron Diffraction: Structure and Dynamics of Free Molecules and Condensed Matter (Moscow, Fizmatlit, 2013)

[2] J.S. Baskin, A.H. Zewail, C.R. Phys. 15, 176 (2014)

[3] B.N. Mironov, V.O. Kompanets, S.A. Aseyev, A.A. Ischenko, O.V. Misochko, S.V. Chekalin, E.A. Ryabov, JETP Lett. 103, 531 (2016)

[4] K. Ishioka, M. Kitajima, O.V. Misochko, J. Appl. Phys. 103, 123505 (2008)

[5] S.V. Andreyev, S.A. Aseyev, V.N. Bagratashvili, N.S. Vorob'ev, A.A. Ischenko, V.O. Kompanets, A.L. Malinovsky, B.N. Mironov, A.A. Timofeev, S.V. Chekalin, E.V. Shashkov, E.A. Ryabov, Quantum Electronics 47(2), 116 (2017)

[6] D.S. Badali, R.Y.N. Gengler, R.J.D. Miller, Struct. Dynamics 3, 034302 (2016) 